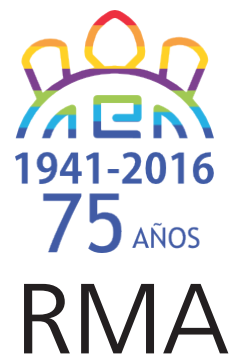

Arqueología

\title{
Implicancias experimentales para una tecnología extractiva de crustáceos de agua dulce mediante el uso de alfarería. Lago Lácar, Patagonia noroccidental (Argentina)
}

\author{
Implications for experimental technology extractive \\ freshwater crustaceans using pottery. Lácar lake, \\ northwest Patagonia (Argentina)
}

\begin{abstract}
Alberto E. Pérez* y Verónica Schuster**
* Universidad Maimónides (CEBBAD - Fundación Azara) y Laboratorio de Arqueología y Etnohistoria, Secretaría de Planificación y Desarrollo Sustentable, Municipalidad San Martín de los Andes. Rudecindo Roca y Rivadavia (8370). San Martín de los Andes, Neuquén, Argentina. E-mail: arqueo.meliquina@gmail.com ** IDEAus, Centro Nacional Patagónico (CENPAT - CONICET). Boulevard Brown 2915 (9120). Puerto Madryn, Chubut, Argentina. E-mail: veroschus@hotmail.com
\end{abstract}

\begin{abstract}
Resumen
En este trabajo se propone un estudio experimental que tiene por objetivo determinar si recipientes cerámicos sumergidos bajo el agua pueden resultar útiles y eficientes para capturar crustáceos en los lagos de la Patagonia noroccidental argentina. Los resultados obtenidos han demostrado que vasijas experimentales similares a las arqueológicas encontradas bajo el agua, son herramientas eficientes como trampas para la captura de crustáceos. Además, a partir del hervido de los mismos se pudieron extraer y concentrar todos los nutrientes que fueron transferidos a un líquido de cocción o sopa. De esta experimentación resultó un alimento palatable y nutritivo a un bajo costo de aprovisionamiento y procesamiento a partir del uso de la alfarería en ambas etapas, es decir para la obtención o extracción de crustáceos y en su posterior cocción. Se propone así que las actividades del tipo extractivas -además de las tradicionalmente asignadas a la tecnología cerámica como cocción, almacenamiento y/o transporte- puede ser una de las causas de la gran cantidad y diversidad de fragmentos cerámicos recuperados en contextos subacuáticos y en las playas del lago Lácar.
\end{abstract}

Palabras clave: Alfarería; Patagonia noroccidental; lago Lácar; crustáceos; experimentación.

\begin{abstract}
This paper presents an experimental and actualistic study that aims to determine if ceramic containers submerged under water can be useful and efficient tools for catching crustaceans in the lakes of the region under study. The results have shown that experimental vessels, similar to the archaeological vessels found underwater, are efficient traps for catching shellfish. Furthermore, from the boiling of these, all the nutrients could be extracted and concentrated which were then transferred to a cooking liquid or soup. From this procedure, a palatable and nutritious food was obtained at low cost of sourcing and processing, through the use of pottery in both stages: for obtaining or extracting crustaceans and its later cooking. In this manner it is proposed that extractive activities -in addition to those traditionally assigned to ceramic technology such as cooking, storage and/or transportation can be the cause of the large quantity and diversity of ceramic fragments found in underwater environments and the shores of lake Lácar.
\end{abstract}

Keywords: Pottery; northwest Patagonia; Lácar lake; crustaceans; experimentation.

\section{Introducción}

El área de estudio de este trabajo comprende la cuenca del lago Lácar que ha sido incluida recientemente dentro del "Área Arqueológica de la Cuenca Binacional del Río Valdivia, Sector Oriental Cordillerano" (Pérez 2015) (Figura 1). En sus orillas se han identificado sitios arqueológicos de tipo campamentos residenciales y/o logísticos a cielo abierto en los cuales el material predominante es la alfarería. Específicamente en el sitio arqueológico isla Santa Teresita (Figura 1) se ha recuperado gran cantidad y diversidad de alfarería, parte de esta asociada a los vestigios más duraderos de crustáceos Samastacus sp. Estudios previos en otras áreas arqueológicas cercanas -como Lago Meliquina (Pérez 2010)- habían ya dado 
cuenta de una importante representación de esta tecnología frente a otras tradicionalmente predominantes y abundantes en el registro arqueológico patagónico como el material lítico y óseo.

La particularidad de la cuenca Lácar es que además de registrarse abundante cerámica en sus costas se han realizado hallazgos aislados subacuáticos de fragmentos y vasijas semi-completas por debajo de línea actual de la costa y hasta los 12 metros de profundidad. La recurrencia de este tipo de materiales bajo el agua puede corresponderse y/o provenir de distintas causas naturales (ej. procesos gravitacionales, inundaciones etc.) como así también a consecuencia de diferentes actividades o conductas implementadas por el hombre en el pasado (ej. almacenaje, descarte, pérdida, entre otras). En este caso puntual, nos interesa explorar las actividades humanas potencialmente responsables de la presencia de alfarería en contextos subacuáticos así como las probables conductas que derivaron en una mayor tasa de depositación de fragmentos cerámicos en contextos arqueológicos costeros en esta cuenca. Entre las hipótesis que esgrimimos al respecto se señalan:

1- que estas piezas puedan haber permanecido in situ como consecuencia de ocupaciones humanas en el pasado, cuando las playas o la línea de costa habría estado más baja que en la actualidad;

2- que en contextos ceremoniales o rituales estas vasijas y su contenido conformaron ofrendas depositadas en el lago (Gröeber y Palavecino 1928); 3- que estos contenedores pudieron ser extraviados al momento de ser semi-sumergidos con la finalidad de enfriar o conservar algún tipo de alimento y/o bebida;

4- o bien, que pudieron ser implementadas en otros usos no tradicionales como por ejemplo su uso como trampas para obtener crustáceos.

Esta última hipótesis es la que se propone informar y contrastar en este trabajo, sin embargo, se entiende que todas las anteriores expresadas pueden también haber sido causantes de la abundancia y diversidad cerámica en estos sitios arqueológicos lacustres.

Hasta el momento la tecnología cerámica no había sido relacionada con potenciales actividades extractivas o de procuramiento primario de alimentos entre las sociedades cazadoras-recolectoras de la Patagonia Argentina. Tradicionalmente, su rol ha sido circunscripto a funciones domésticas vinculadas con actividades de contención o almacenamiento, procesamiento, cocción y/o transporte de diferentes tipos de recursos (Aldazabal et al. 2008-2009; Aldazabal y Eugenio 2009; Pérez 2011; Schuster 2014).

En el área de estudio de este trabajo, la alfarería es abundante y generalmente monócroma color pardo. A grandes rasgos los cuerpos son globulares, las bases no son formatizadas (cóncavas), las bocas son restringidas -en ocasiones reforzadas-y presentan cuellos cilíndricos y asas. Estas usualmente son verticales, en cinta, de sección circular u ovalada con adelgazamiento longitudinal o lenticular que fueron "remachadas" en el cuerpo y/o cuello-borde de las piezas. En ocasiones estas asas presentan apéndices al pastillaje (protúberos) y modelados anfibiomorfos abstractos y antropomorfos. La decoración aparece en algunos casos como improntas negativas de hojas y por medio de incisiones lineales paralelas en la intersección del cuerpo-cuello en forma directa, o bien sobre bandas de engrosamiento al pastillaje.

Las formas representadas en sitios a cielo abierto como en estratigrafía son principalmente jarros y ollas de diversos tamaños que se caracterizan por su boca de tendencia cerrada, ya que los grupos morfológicos abiertos -como los cuencos- están muy escasamente representados. Entre las piezas subacuáticas se destacan las formas con cuello y boca restringida, principalmente jarras y ánforas. Estas últimas -las ánforas- solo han sido observadas hasta el momento en este tipo de contexto.

Los lagos andinos norpatagónicos cuentan con una gran diversidad y abundancia de recursos potencialmente aprovechables por el hombre. Entre ellos, y a los fines del presente trabajo, se señalan dos especies de crustáceos: un cangrejo del género Aegla sp. (Leach 1820) -conocido como "Pancora"- y el camarón Samastacus spinifrons (Philippi 1882) que da nombre a un lago de la región en idioma mapuche como "Machonico" (Koessler-Ilg 1962). En este trabajo se propone que la alfarería podría haber sido empleada en el pasado para aprovechar y optimizar recursos tan abundantes, predecibles y resistentes a la sobreexplotación como son los crustáceos, no sólo mediante las prácticas o técnicas culinarias (ej. guisado, hervido, etc.) sino también como una tecnología propicia para la captura de estas presas mediante el trampeo.

Específicamente, en el contexto de intensificación que caracteriza al Período Alfarero Temprano en el área de estudio (Pérez 2015), la cerámica pudo resultar tanto un medio útil para procesar y almacenar como así también para extraer y obtener alimentos. La intensificación aquí se postula en un incremento en la variedad de ítems explotados que redunda en el aumento de la productividad total por unidad de tiempo y espacio (Boresup 1965 en Medina y Prates 2014; Broughton 1999) a partir de un incremento de la capacidad de subsistencia promovido por cambios en las condiciones tecnológicas (Medina y Prates 2014).

Para contrastar esta hipótesis -empleo de vasijas cerámicas como trampas para crustáceos- proponemos un estudio experimental y actualístico que tiene por objetivo determinar si este tipo de recipientes cerámicos sumergidos bajo el agua pueden resultar útiles y eficientes para la captura de cangrejos y/o camarones nativos en 


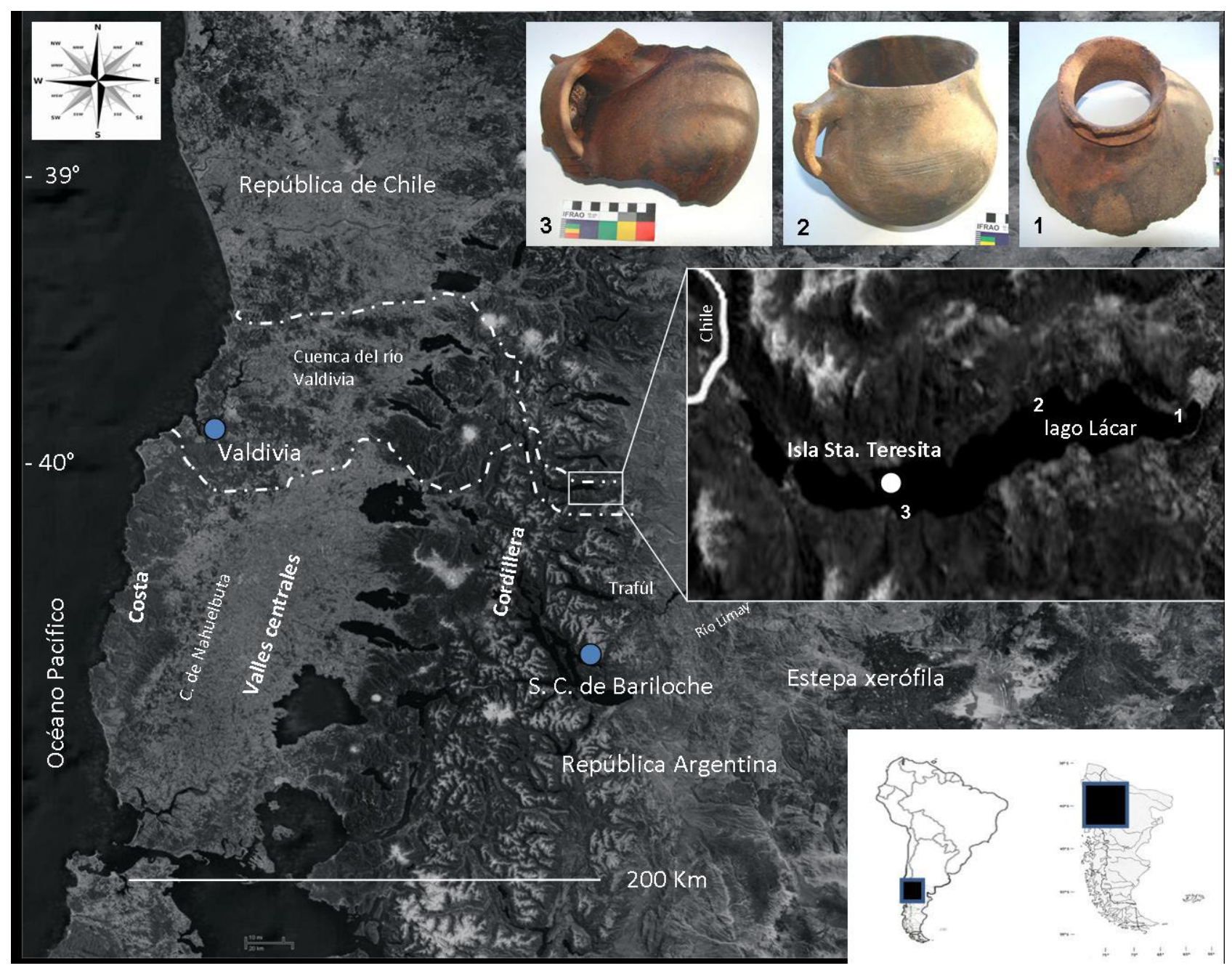

Figura 1. Área de estudio, fotografías de piezas cerámicas recuperadas en contexto subacuático con su respectiva ubicación en el mapa ( $N^{\circ}$ 1, $2,3)$ y detalle de la cuenca del lago Lácar donde se señala el sector de trampeo de crustáceos con vasijas experimentales (isla Santa Teresita).

Figure 1. Study area, pictures of pottery recovered in underwater context (numbers 1, 2,3) and Lácar lake detail of the area with crustacean traps made with experimental vessels (Santa Teresita island).

los lagos de la región. La base de esta propuesta no solo está relacionada con los hábitos cavícolas de estas especies sino también con las ventajas nutricionales que los hacen muy propicio para el consumo humano más allá de la escasa información actual -tanto biológica como arqueológica- respecto de este tema.

\section{Breve reseña de los recursos en el área de estudio}

El bosque norpatagónico presenta una gran variedad de recursos faunísticos. La mayor cantidad de especies corresponde a animales de pequeño porte, ya que los de mayor tamaño se presentan en bajo número, dispersos y presentan hábitos eto-ecológicos singulares lo que los hacen elusivos y muy susceptibles a la sobreexplotación. Los ciclos reproductivos de los grandes herbívoros son más lentos (Eisemberg 1989) por lo que este factor se potencia por su baja abundancia y densidad e incrementa el peligro de sobreexplotación rápida, al menos a nivel local (Hayden 1981; Stahl 1995). En contraste, la población de especies de menor porte son más abundantes, densas y su renovación biológica es rápida (Hayden 1981; Stahl 1995).

Entre los recursos que pueden ser recolectados masivamente se pueden mencionar las semillas, los frutos, los insectos, los animales pequeños, los peces, los moluscos y crustáceos. En estos alimentos la unidad de comparación no es la presa individual sino el volumen de captura masiva. Es por esto que los recursos que se pueden colectar en forma masiva se constituyen en presas de alto ranking (Pérez et al. 2008), ya que hay que tener en cuenta la utilidad "post encuentro" a partir de la alta densidad de estas presas en el paisaje (Madsen y Schmitt 1998). Particularmente, nos interesa para este trabajo abordar dos especies de crustáceos de agua dulce que son abundantes y predecibles en la región: el cangrejo Aegla y el camarón Samastacus.

La Aegla es un género restringido en las regiones centrales y australes de América del sur, específicamente habitan en cuerpos acuáticos de agua dulce entre el nivel marino y los 


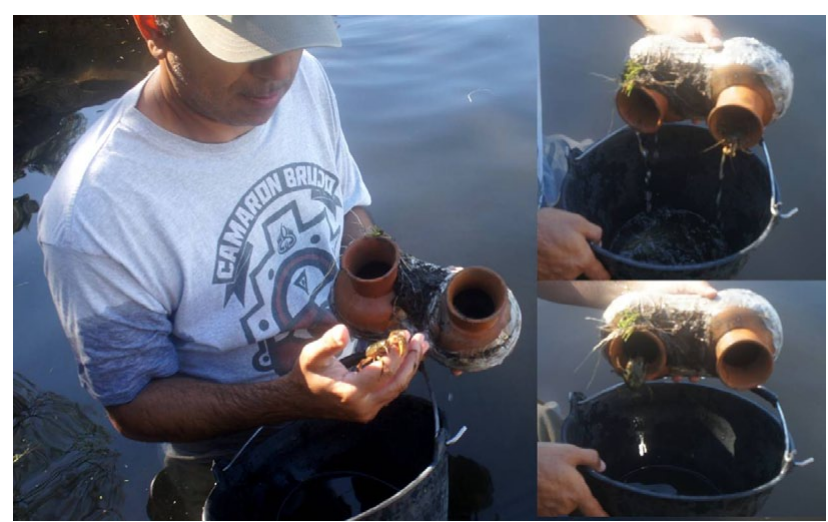

Figura 2. Extracción de paquetes de vasijas experimentales para e registro y conteo de los especímenes capturados.

Figure 2. Experimental vessels packages recovery for recording and counting of captured specimens.

4.500 msnm al sur de Brasil, Bolivia, Paraguay, Uruguay, gran parte de Argentina y Chile. Son especies que recorren el bentos de los cuerpos de agua en los que habitan en búsqueda de alimento y ocultándose bajo rocas del lecho, en cuevas o grietas. Su dieta es omnívora -desde materia vegetal a insectos, moluscos, peces, renacuajos, etc.- y su fisionomía resulta similar a los langostinos, teniendo el abdomen parcialmente oculto por debajo del tórax. El Samastacus habita en ambientes de agua dulce del sudoeste de América del Sur. Se trata de un crustáceo de fondo, de alimentación oportunista, generalista, politrófico. Tanto en lo que respecta al sabor como a la textura, su carne es de excelente calidad.

En el área de estudio ambos crustáceos son abundantes hoy en día a pesar de ser depredados por especies introducidas para la pesca deportiva como los salmónidos. Aunque no podemos afirmar que una especie pudo ser más valiosa que la otra como alimento ya que no conocemos registros de su abundancia en el pasado, existe un consenso entre los especialistas de que Aegla sp. suele ser más abundante en un mismo ambiente que el género Samastacus sp. (Luquet 2015, Com. Pers). Estas especies presentan características singulares para su aprovechamiento: el camarón tiene una porción carnosa comestible en su segmento abdominal trasero y órganos y fluidos en su segmento cefálico, mientras que el cangrejo presenta una porción carnosa más reducida parcialmente cubierta por su tórax, donde se encuentran además los órganos y fluidos.

Por lo expuesto, los crustáceos habrían sido recursos previsibles, localizados, abundantes, y por lo tanto, confiables y de rápida renovación para su aprovechamiento. Su aporte a la dieta -si bien en apariencia bajo cuando se estima por unidad- debe ser considerado y revalorizado en base a los bajos costos de obtención y a su volumen como producto susceptible de ser capturado en forma masiva y por medio de trampas.
El uso de trampas para la captura de recursos dulceacuícolas en la cuenca valdiviana está ampliamente representado en el registro etnohistórico y arqueológico (Álvarez et al. 2013). El lago Lácar, naciente de esta cuenca, no es la excepción ya que presenta descripciones del uso trampas para la pesca (Cox 1862-1863) incluyendo corrales de piedra y estructuras vegetales que conducen a las potenciales presas -ej. cangrejos, peces, etc- hacia un embudo o "nasa", denominadas "Llolles" en idioma nativo (Álvarez et al. 2013). Si bien en la región el uso de trampas está muy documentado para la fauna lacustre y terrestre, no hay referencias que den cuenta de la implementación de cerámica en estas tareas.

\section{Metodología experimental}

\section{a- Trampeo de crustáceos}

Para estas tareas se emplearon cuatro vasijas experimentales de cuerpo globular, cuello cilíndrico y boca restringida, características que fueron replicadas de algunas de las piezas arqueológicas recuperadas en contextos subacuáticos y en el valle del lago Lácar así como también de la localidad arqueológica cercana Lago Meliquina (Pérez 2011, 2015).

Estas piezas se elaboraron a partir de la técnica de rodete, con arcillas locales y se estandarizó su capacidad/volumen en $750 \mathrm{~cm}^{3}$ (Figura 2). Con ellas se armaron paquetes 0 grupos de dos vasijas (que llamaremos A y B) que fueron colocadas entre los 0.60 y 1.00 m de profundidad en un sector rocoso y un sector fangoso de la isla Santa Teresita en la cuenca Lácar (Figura 1). Luego de un período de tres días, las vasijas fueron retiradas del agua y su contenido fue colocado en un recipiente plástico para proceder al registro de la presencia de fauna acuática, la identificación taxonómica y la cuantificación de los especímenes capturados (Figura 2). Inmediatamente después de este paso se colocaron nuevamente las vasijas vacías bajo el agua; proceso que fue repetido cinco veces. Finalmente, cabe mencionar que las capturas fueron posteriormente liberadas en sectores distantes a $5 \mathrm{~km}$ del lugar de trampeo con la intención de evitar la reocupación de las vasijas por parte de los mismos individuos.

\section{b- Cocción de crustáceos}

Se utilizó una vasija experimental que replica al grupo morfológico más recurrente en los sitios arqueológicos del área de estudio (Pérez 2011, 2015) y particularmente, similar a una de las piezas recuperadas en contexto subacuático del lago Lácar (Figura 1: fotografía 1). Esta es de forma globular, boca levemente restringida, con un asa lateral y una capacidad o volumen aproximado de $1.000 \mathrm{~cm}^{3}$. Fue manufacturada a partir de rollos o rodetes con arcillas de provisión local y cocinada en fogón abierto (Pérez et al. 2015).

Para preparar el caldo o sopa se colocaron en el fondo de esta vasija 10 crustáceos de la especie Aegla sp. 


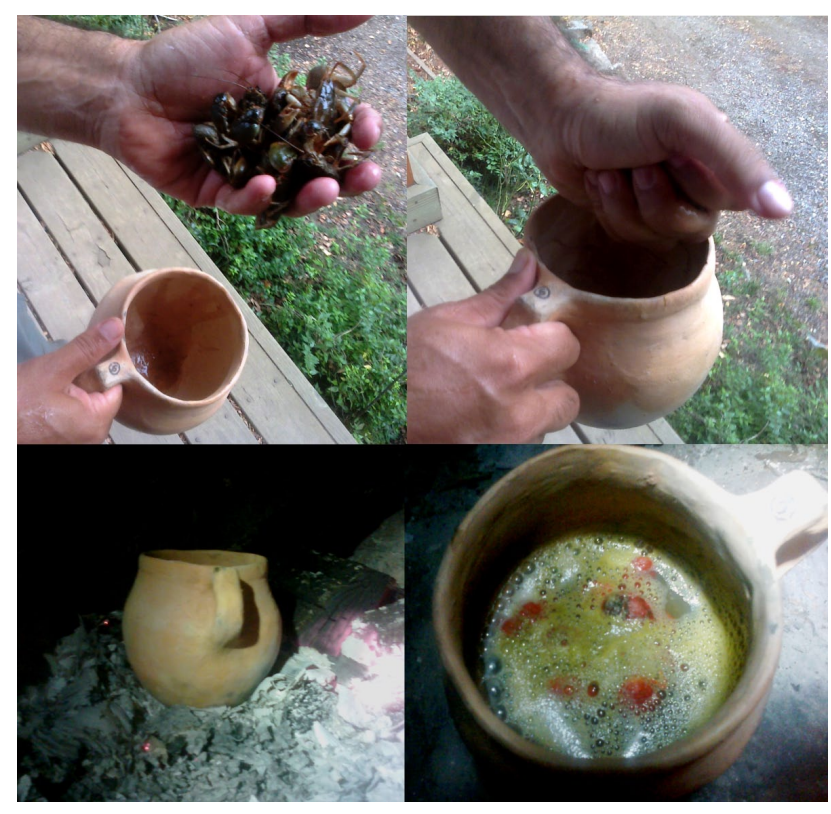

Figura 3. Cocción de crustáceos en una pieza cerámica experimental.

Figure 3. Crustaceans cooked in experimental pottery vessels

-previamente machacados o triturados a mano- que fueron cubiertos con agua hasta $3 / 4$ partes de la capacidad máxima del contenedor. Posteriormente, este fue colocado sobre brasas hasta llegar al punto de hervor a partir del cual se prolongó la cocción durante 30 minutos (Figura 3). El procedimiento completo -encendido del fuego, preparación y cocción de los crustáceos- fue menor a una hora reloj.

\section{Resultados obtenidos}

\section{Trampeo}

Estas tareas -que se llevaron adelante colocando los paquetes o grupos de vasijas bajo el agua- es un proceso cuyo costo cinegético podemos caracterizar como bajo ya que se requiere de solo una breve caminata de entre 5 a 10 metros de la playa hasta alcanzar profundidades de $1 \mathrm{~m}$ donde el lago presenta un sustrato con cualidades adecuadas para la colocación de las trampas.

El primer muestreo tuvo resultado positivo en una sección rocosa y negativo en un sector fangoso con vegetación, sustratos en que fueron depositados los paquetes $A$ y $B$, separados por solo $2 \mathrm{~m}$ de distancia. Teniendo en cuenta este resultado y contemplando los aspectos etoecológicos de las potenciales presas, se decidió continuar los siguientes cuatro muestreos sólo en el sector rocoso donde estos crustáceos suelen buscar y ocupar refugios naturales. Se obtuvieron así capturas en todos ellos y no se registraron fuga de presas durante las extracciones de las trampas. Esta tarea se realizó mediante una sencilla y rápida recolección manual de los especímenes (Figura 2).

El género ampliamente representado en los muestreos fue el cangrejo $(\mathrm{N}=18)$ y sólo en un único caso se registró camarón (Figura 4). Todos los individuos capturados corresponden a machos adultos, es decir a los más grandes en tamaño. Posiblemente el carácter restringido de las vasijas utilizadas sea responsable de la competencia intraespecífica (machos adultos) e interespecífica por un refugio (Pancora es una especie más agresiva y exitosamente más competitiva que Samastacus). Esta observación puede resultar un sesgo en el muestreo pero no afecta de ninguna forma el objetivo y los resultados del experimento que tenían por finalidad testear el uso de las vasijas cerámicas como un modo eficaz de trampeo para crustáceos.

\section{Cocción}

Durante la cocción de los cangrejos se observó que estos modificaron la coloración de su caparazón tornándose anaranjados debido a que las temperaturas alcanzadas

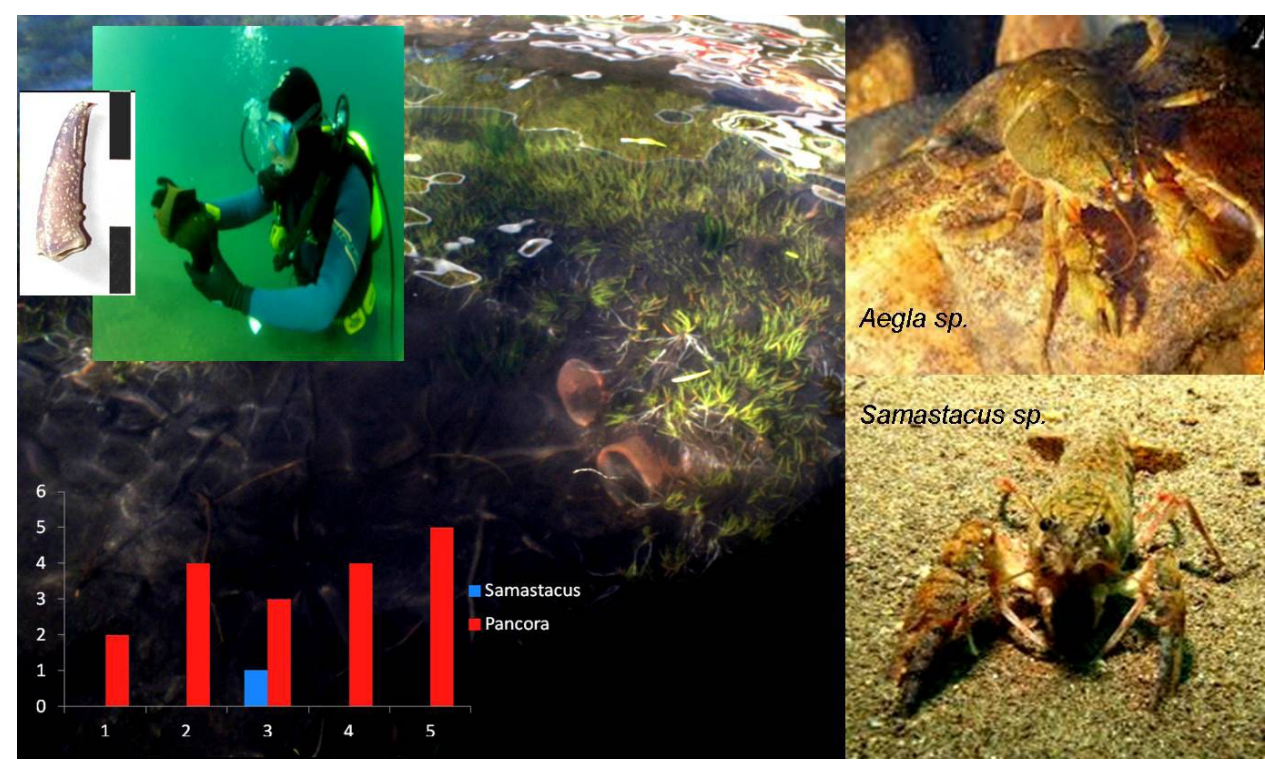

Figura 4.Grafico con el número de capturas por taxón obtenidos en los muestreos; imágenes de las especies de crustáceos mencionadas en el trabajo; fragmento cerámico en contexto subacuático y segmento de pinza de Samastacus del sitio isla Santa Teresita. De fondo, vista de algunas piezas cerámicas experimentales usadas como trampas bajo el agua.

Figure 4. Graphic with number of taxa obtained in samples, pictures of specimens mentioned in this work, pottery fragment in underwater context and of a Samastacus clipper from Santa Teresita Island's archaeological site. In the background, photography of some experimental vessels used as traps in underwater. 


\begin{tabular}{ccc}
\hline Especie & Carne de quelas & Carne de abdomen \\
\hline Samastacus spinifrons & 15,2 & 17,2 \\
Parastacus pugnax & 15,2 & 14,2 \\
Parastacus nicoleti & 18,6 & 15,1 \\
\hline
\end{tabular}

Tabla 1. Análisis de laboratorio de Aegla sp. colectadas en el Río Chimehuín, Neuquén (Hualde 1997). Referencias: *Nitrógeno por método semi micro Kjeldhal; Lípidos por método Soxhlet; humedad por secado a $105^{\circ} \mathrm{C}$; cenizas por calcinación a $600^{\circ} \mathrm{C}$; E.L.N. por diferencia; energía a razón de 5.64, 9.44 y 4.11 kcal por g de proteínas, lípidos y carbohidratos respectivamente.

Table 1. Laboratory analysis of Aegla sp. collected in the Chimehuin river, Neuquén (Hualde 1997). References: * Nitrogen by Kjeldhal semi micro method; Lipids by Soxhlet method; moisture by drying at $105^{\circ} \mathrm{C}$; ash by calcination at $600{ }^{\circ} \mathrm{C}$; E.L.N by difference; energy at a rate of 5.64, 9.44 and $4.11 \mathrm{kcal}$ by $4.11 \mathrm{~g}$ of protein, lipids and carbohydrates respectively.

permitieron la formación de carotenoide astaxantina que se libera a partir de la cromoproteína verde-parduzca que poseen estas especies.

De este proceso se obtuvo una sopa espesa color amarillento, de aroma y sabor similar al camarón (Figura 3). Es importante destacar que el hervido de los crustáceos machacados permitió extraer, concentrar y transferir todos los nutrientes (fluidos y carne) al líquido de cocción. En suma, se obtuvo un producto alimenticio, palatable y nutritivo con un bajo costo de aprovisionamiento y procesamiento a partir del uso de alfarería en ambas etapas, es decir, tanto para la obtención o extracción por trampeo de los crustáceos como para su cocción.

Los análisis químicos realizados a esta especie de cangrejo (Tabla 1) como al camarón (Tabla 2) permiten sustentar el valor nutricional de los mismos.

\section{Discusión}

De manera general se puede mencionar que de las tareas de trampeo y de la cocción experimental pudo con muy poco esfuerzo asegurarse alimentos muy nutritivos para la dieta humana. Específicamente respecto a la potencial cocción de crustáceos en vasijas cerámicas y su posterior consumo, debe tenerse en cuenta que el valor energético del cangrejo Aegla y el camarón Samastacus es similar al pescado y que presentan casi la mitad de sus proteínas. Respecto a esta última especie se destaca que su carne es de excelente calidad -tanto en textura como en sabor- y que presenta un elevado contenido de proteínas, resultando análoga al contenido en carnes de pollo, merluza, ancas de rana y algunos moluscos marinos comestibles (Urbanski et al. 2005). Estos datos son importantes si tenemos en cuenta que estos recursos son altamente proteicos y por lo tanto, esenciales para una dieta equilibrada.

\begin{tabular}{ccc}
\hline & Base Húmeda & Base Seca \\
\cline { 2 - 3 } Proteínas (\%) & 12.1 & 53.2 \\
Extracto Étereo (\%) & 2 & 8.7 \\
EL N (\%) & 2.5 & 11.1 \\
Cenizas (\%) & 6.1 & 27 \\
Humedad (\%) & 77.3 & 0 \\
Energía bruta (Kj/g) & 3.1 & 13.5 \\
Fósforo (\%) & 0.3 & 1.34 \\
\hline
\end{tabular}

Tabla 2. Resultados de análisis químicos de Samastacus sp. colectados en el río Traful (Neuquén) y estudiados en cautiverio. Los parámetros húmedos/secos se basan en el total registrado para abdomen y cefalotórax. (Tomado y modificado de Urbanski et al. 2005).

Table 2. Results of chemical analysis of Samastacus sp. collected in the Traful river (Neuquén) and studied in captivity. The wet/dry parameter based on the total recorded for abdomen and cephalothorax. (taken and modified Urbanski et al. 2005).

Las características del registro arqueológico de estos sitios lacustres no han permitido una buena conservación de este tipo de recursos. Hasta el momento, solo se han identificado los restos más duros como son las pinzas del genero Samastacus (Pérez 2015, Com Pers).

La alfarería cobra así nuevamente un status importante en la ampliación exponencial de nutrientes en un mismo territorio. La incorporación de esta tecnología en el ámbito boscoso y lacustre se constituye como una herramienta fundamental para la intensificación de los recursos para la subsistencia humana exitosa en este tipo de ambiente. Al respecto, una de las ventajas del empleo de la tecnología cerámica en grupos cazadores-recolectores que explotan ambientes con animales de hábitos dispersos es lo que se denomina como oil production, es decir la extracción de grasas animales y aceites vegetales que se lograría eficazmente a partir de la cocción indirecta (Hoopes 1995; Sassaman 1995). De esta manera la exposición indirecta al fuego aumenta el valor nutricional y disminuye la toxicidad de determinado grupo de alimentos. Al mismo tiempo, el hervor prolongado logra reducir la fibra de determinados vegetales aumentando la concentración de carbohidratos susceptibles de más rápidamente absorbidos durante la digestión (versus los alimentos tostados y/o asados), esteriliza los alimentos y facilita la extracción de grasas (Sassaman 1995).

Las actividades extractivas -como la propuesta en este trabajo- además de las ya tradicionalmente asignadas a esta tecnología pueden ser una de las causas de la gran cantidad y diversidad de fragmentos y piezas cerámicas parcialmente completas en las costas y en las profundidades del lago Lácar. Los resultados experimentales han demostrado que vasijas similares a las arqueológicas encontradas bajo el agua son herramientas potencialmente eficientes como trampas para la captura de crustáceos. La experimentación realizada nos permite plantear como hipótesis que algunas de las 
piezas encontradas en contextos subacuáticos podrían corresponderse con trampas perdidas y/o abandonadas que podrían haber sido utilizadas eficientemente. Por otra parte, la incorporación de estos recursos abundantes, predecibles y casi invulnerables a la sobreexplotación habría permitido garantizar una ingesta energética y proteica con un bajo costo cinegético en la dieta de las poblaciones locales en el pasado.

A futuro se espera continuar esta línea de trabajo con estudios químicos en el interior de las pastas cerámicas (ej. ácidos grasos) para determinar probables elementos traza de estos crustáceos como otra línea de evidencia que permitirá explorar el procesamiento y/o almacenamiento de este tipo de recursos en los contenedores cerámicos así como evaluar y discutir su importancia en la dieta humana.

San Martín de los Andes, 15 de Junio de 2015

Agradecimientos: Al Dr. Carlos Luquet (INIBIOMA, CEAN-CONICET. Junín de los Andes, Neuquén) por su asesoramiento en los valores nutricionales de las dos especies mencionadas en este trabajo, a la ceramista Iris Otaño por la confección de la pieza experimental utilizada para la cocción de crustáceos y a Luis Hermann quién elaboró las vasijas utilizadas como trampas.

\section{Bibliografía}

Aldazabal, V., Silveira, M., \& Micaelli, A. (2008-2009). La cerámica del sitio alero Las Mellizas, lago Traful, Provincia de Neuquén. Anales de Arqueología y Etnología, 63-34, $1-24$.

Aldazabal, V. B., \& Eugenio, E. O. (2009). Entre el fuego y el juego. La cerámica del sitio Rincón Chico 2/87. En E. A. Crivelli Montero, M. A. Fernández, \& M. S. Ramos (Eds.), Arqueología de rescate en Rincón Chico, provincia del Neuquén (pp. 163-186). Buenos Aires: Dunken.

Álvarez, R., Munita, D., Hernández, J., Barón, A., \& Gálvez, D. (2013). Antecedentes etnográficos e históricos de la pesca con nasas (Llolles) en el Centro Sur de Chile. Revista Austral de Ciencias Sociales, 25, 5-21.

Broughton, J. M. (1999). Resource depression and intensification in Late Holocene San Francisco Bay: evidence from the Emeryville Shellmound. Berkeley: University of California.

Cox, G. (1999) [1862-1863]. Viaje a las rejiones septentrionales de la Patagonia. Buenos Aires: Elefante Blanco.

Eisenmberg, J. F. (1989). Mammals of the Neotropics, Vol. 1. The Northern Neotropics: Panamá, Colombia, Venezuela, Guyana, Suriname, French Guiana. Chicago:
University of Chicago Press.

Gröeber, P., \& Palavecino, E. (1928). Un Ngillatum en el lago Lakar. GAEA. Anales de La Sociedad Argentina de Estudios Geográficos, 3(1), 291-314.

Hayden, B. (1981). Research and development in the Stone Age: technological transitions among huntergatherers. Current Anthropology, 22(5), 519-548.

Hoopes, J. (1995). Interaction in hunter and gathering societies as a contex for the emergence of pottery in the Central American Isthmus. En The emergence of pottery. Technology and innovation in ancient societies (pp. 185198). Washington and London: Smithsonian Institute.

Hualde, P. (1997). Análisis de Laboratorio de Aegla sp. N54-3. Centro de Ecología Aplicada de Neuquén (CEAN), Junín de los Andes.

Koessler-llg, B. (1962). Tradiciones Araucanas. Tomo I. Buenos Aires: Universidad de La Plata.

Madsen, D., \& Schmitt, D. (1988). Mass Collecting and the Diet Breadth Model: A Great Basin Example. Journal of Archaeological Science, 25, 445-455.

Medina, M. E., \& Prates, L. (2014). El estudio arqueológico de los procesos de intensificación. Casos de Europa y América del Sur. Comechingonia, 18(2), 9-15.

Pérez, A. (2015). El registro arqueológico de la Cuenca Binacional del río Valdivia. La integración de su fuente, el lago Lácar, sector oriental cordillerano. En A. Nicoletti, A. Núñez, \& P. Núñez (Eds.), Araucanía-Norpatagonia, III, Discursos y representaciones de la materialidad (pp. 144-188). Bariloche: Universidad Nacional de Río Negro.

Pérez, A. E. (2011). Algunas reflexiones sobre la alfarería del Centro Sur de Chile y Ambientes Lacustres Precordilleranos de la Patagonia Septentrional Argentina. En P. Floria Navarro \& W. Delrio (Eds.), Cultura y Espacio. Araucanía-Norpatagonia (pp. 293-315). Bariloche: Universidad Nacional de Río Negro.

Pérez, A. E. (2010). La Localidad Arqueológica "Lago Meliquina", Dto. Lácar, Neuquén. El registro arqueológico del interior y borde de bosque en Norpatagonia. En Actas y Memorias del XVII Congreso Nacional de Arqueología Chilena (2006) (pp. 1515-1528). Valdivia.

Pérez, A. E., Giesso, M., \& Glascock, M. D. (2015). Fuentes de aprovisionamiento y uso de obsidianas en el ámbito boscoso y lacustre andino norpatagónico (Provincia del Neuquén, Argentina). Intersecciones en Antropología, Volumen Especial(2), 17-26.

Pérez, A. E., Cirigliano, N., López, L., \& Batres, D. (2008). Disponibilidad de materias primas líticas en la localidad 
arqueológica Meliquina, Parque Nacional Lanín, Provincia de Neuquén, República Argentina. Revista Werken, 10, 127-145.

Rudolph, E., Martínez, F., \& Retamal, A. (2010). Cultivo de camarón de río Samastacus spinifrons: ¿una nueva alternativa para la diversificación de la acuicultura chilena? Latin American Journal of Aquatic Research, 38(2), 254.

Rudolph, E., de la Fuente, L., Van Hasselt, M. E., Díaz, A., \& Dumenes, S. (1991). Composición química proximal de los tejidos comestibles y desperdicios de los camarones dulceacuícolas Samastacus spinifrons, Parastacus pugnax y Parastacus nicoleti (Crustácea: Decapoda: Parastacidae). Alimentos, 16(2), 23-29.

Sassaman, K. (1995). The social contradiction of traditional and innovative cooking technlogies in the prehistoric American Southeast. In The emergence of pottery. Technology and innovation in ancient societies (pp. 223-240). Washington and London: Smithsonian Institute.

Schuster, V. (2014). La organización tecnológica de la cerámica de cazadores-recolectores. Costa norte de la Provincia del Chubut (Patagonia Argentina). Relaciones de La Sociedad Argentina de Antropología, 39(1), 203-231.

Stahl, P. (1995). The recovery and interpretation of microvertebrate bone assemblages from archaeological context. Journal of Archaeological Method and Theory, 3, 31-75.

Urbanski, J., Laffitte, L., Ambrosio Espinós, A., Hualde, P., \& Luquet, C. (2005). Cría experimental del camarón de río Samastacus Spinifrons (Philippi 1882) (Decapoda: Parastacide). Crecimiento y reproducción en cautiverio. Ms 